\title{
Time for evidence-based, standardized donor size matching for pediatric heart transplantation
}

Kyle W. Riggs, MD, ${ }^{\mathrm{a}}$ Courtney M. Giannini, BS, ${ }^{\mathrm{b}}$ Nicholas Szugye, MD, ${ }^{\mathrm{c}}$ Jason Woods, PhD, ${ }^{\mathrm{d}}$

Clifford Chin, MD, ${ }^{\mathrm{c}}$ Ryan A. Moore, MD, ${ }^{\mathrm{c}}$ David L. S. Morales, MD, ${ }^{\mathrm{a}}$ and Farhan Zafar, MD

\section{ABSTRACT}

Background: Accurately predicting cardiac size by other body parameters has long been problematic to determine whether a donor heart will serve a given waitlist candidate, yet hundreds of heart donors are turned down annually for size mismatch.

Objectives: We sought to describe how donor body weight parameters are currently utilized in cardiac transplantation and its influence on waitlist outcomes.

Methods: From the United Network for Organ Sharing database, pediatric (age $<18$ years) heart transplant candidates were divided into lower quartile, interquartile, and upper quartile categories based on final maximum acceptable donorcandidate weight ratio (DCW), expressed as percentage. Baseline characteristics and waitlist outcomes, including monthly offers/candidate and survival were compared.

Results: Overall median DCW was 200\% (range, 159\%-241\%). Patients with congenital heart disease had higher DCW than those with cardiomyopathy $(223 \%$ vs $203 \% ; P<.001)$. Number of monthly offers/candidate $(5.0,5.6$, and 7.2 , respectively; $P<.001)$ increased with quartile of DCW. Posttransplant survival was similar amongst the groups (log-rank $P>.05)$. Subgroup analysis of critically ill children showed a waitlist survival advantage in those listed with a $\mathrm{DCW} \geq 200 \%(P<.001)$.

Conclusions: Despite substantial practice variation in acceptable donor weight in pediatric heart transplantation, patients listed with variable DCW had similar posttransplant survival. However, in critically ill patients, higher DCW was associated with greater waitlist survival. Better understanding of the importance of donor weight could reduce practice variability and improve organ use and waitlist outcomes for pediatric cardiac transplant candidates. (J Thorac Cardiovasc Surg 2019;158:1652-60)

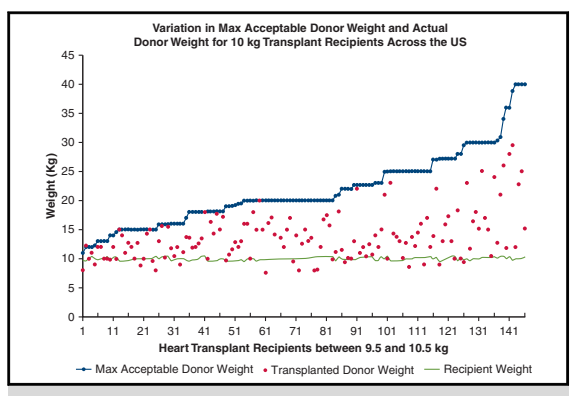

Acceptable maximal donor weight varies greatly across the United States.

\section{Central Message}

There is great variation of acceptable donor-tocandidate weight ratio in pediatric heart transplant. A higher ratio benefits critically ill children but uniformity in listing practices should be sought.

\section{Perspective}

Significant variation in pediatric heart transplantation listing practices influences organ allocation and time on waitlists. There appears to be no nationwide standard for acceptable weight. With better justification and measurements for donor-to-recipient size matching, a more equal system could improve waitlist and posttransplant outcomes.

See Commentaries on pages 1661 and 1663.
Physicians have long sought to estimate the size of the human heart based on other anthropometric factors, even before heart transplantation was feasible. ${ }^{1}$ Although the vast majority of studies come from adults, no sufficient correlating factor has been found between heart size and

From the Divisions of ${ }^{\mathrm{a}}$ Cardiothoracic Surgery, ${ }^{\mathrm{c}}$ Pediatric Cardiology, and ${ }^{\mathrm{d}}$ Pulmonary Imaging Research, Cincinnati Children's Hospital, Cincinnati, Ohio; and ${ }^{\mathrm{b}}$ College of Medicine, University of Cincinnati, Cincinnati, Ohio.

Received for publication Jan 22, 2019; revisions received June 10, 2019; accepted for publication June 14, 2019; available ahead of print July 26, 2019.

Address for reprints: Kyle W. Riggs, MD, Division of Cardiothoracic Surgery, Cincinnati Children's Hospital, 3333 Burnet Ave, MC 2013, Cincinnati, OH 45229

(E-mail: kcriggs00@gmail.com).

$0022-5223 / \$ 36.00$

Copyright (c) 2019 by The American Association for Thoracic Surgery

https://doi.org/10.1016/j.jtcvs.2019.06.037 other measurements. ${ }^{2,3}$ In pediatrics, estimating heart size is further confounded by a growing patient and a variety of pathologies (dilated cardiomyopathy vs single-ventricle congenital heart disease [CHD]). Despite the lack of validation of a correlation between heart size and anthropometric factors, transplant centers continue to mostly use patient weight when determining whether a donor heart is of suitable size for an individual heart transplant candidate, and

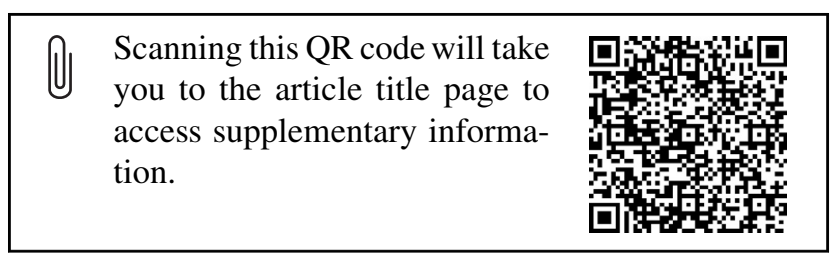




\section{Abbreviations and Acronyms \\ $\mathrm{CHD}=$ congenital heart disease \\ $\mathrm{DCW}=$ donor-candidate weight ratio \\ $\mathrm{ECMO}=$ extracorporeal membranous oxygenation \\ UNOS $=$ United Network for Organ Sharing \\ $\mathrm{VAD}=$ ventricular assist device}

this measure is what is reported to United Network for Organ Sharing (UNOS). Several studies have shown that oversizing or undersizing a donor heart does not adversely affect survival if the donor heart can be made to appropriately fit in the chest cavity. Despite this, there continues to be significant practice variation in how US transplant centers utilize weight criteria for recipient candidate waitlisting. ${ }^{4,5}$ Prior studies have used $<80 \%$ of the transplant candidate's weight for undersizing and more than $250 \%$ of the transplant candidate's weight for oversizing, with very few patients being listed outside of that range. ${ }^{6,7}$ Although waitlist parameters can influence on the number of offers a transplant candidate receives, it has not been shown to affect posttransplant survival. However, the belief among transplant teams that a size mismatch may be associated with poorer outcomes is suggested by the large number of donor refusals $(34 \%)$ for weight mismatch. ${ }^{8}$ Along with other reasons one may refuse an organ offer, only $42 \%$ of pediatric donor hearts were used from 2005 to 2014 . $^{9}$ This seems problematic and an area for focused improvement in donor utilization. Therefore, this study aimed to identify the practice variation in transplant waitlisting across the United States and quantify the influence on waitlist outcomes and posttransplant survival, especially in critically ill heart transplant candidates.

\section{PATIENTS AND METHODS \\ Patient Selection}

After obtaining approval for the study from the Cincinnati Children's Hospital Institutional Review Board, the UNOS database was searched for pediatric patients (age $<18$ years) listed for heart transplantation from 2000 to 2015. Their most recent maximum acceptable donor weight and candidate weight on the waitlist were identified. Patients with missing weight data were excluded from the study $(\mathrm{n}=118[1.6 \%])$. Minimum acceptable weight was clustered around the candidates' actual weight so maximum acceptable weight was analyzed as a percentage of the patient's weight (donor-candidate weight ratio [DCW]). This was used rather than absolute weight difference to account for patients of differing size. Patients were then grouped based on their maximal DCW as lower quartile, interquartile, and upper quartile. Patient indication for transplantation was classified as congenital heart disease, cardiomyopathy, retransplantation, or other. Estimated glomerular filtration rate was by the Schwartz method with $<60 \mathrm{~mL} / \mathrm{min} / 1.73 \mathrm{~m}^{2}$ or pretransplant dialysis classified as renal dysfunction.

\section{Statistical Analysis}

Data are presented as median (interquartile range) when distributed nonnormally and with mean \pm standard deviation when normally distributed. Categorical variables are presented as frequency (\%). Variables with missing data report the $\mathrm{n}$ for that category. Baseline variables at listing and time of transplant and outcomes were compared amongst the groups. An adverse event on the waitlist was defined as removal from the waitlist due to death or decline in health resulting in removal from the waitlist. Fisher exact and $\chi^{2}$ tests were used for categorical variables. Nonparametric tests were used to compare nonnormally distributed data. Kaplan-Meier survival curves with log-rank comparison were recorded for waitlist and posttransplant survival. Cox-regression was used for multivariable analysis of waitlist survival, adjusting for DCW category, patient diagnosis, mechanical ventilation, extracorporeal membranous oxygenation (ECMO), renal dysfunction, and ventricular assist device (VAD) support, all at listing.

\section{Center Data}

The median patient DCW of centers who performed more than 20 transplants within the timeframe of the study was analyzed. The listing DCW and actual transplant DCW are described.

To assess the influence of changes in weight criteria on waitlist outcomes, we created a linear model of the number of changes in weight criteria on the number of days on the waitlist. A change in weight criteria was any absolute change in the maximum weight criteria.

A subgroup analysis focused on mortality in critically ill patients who had CHD, renal dysfunction, and mechanical ventilation, all of which are known risk factors for mortality. ${ }^{10}$ Due to smaller numbers and less variation in listing variables, these patients were divided ad hoc into a Strict DCW and a Liberal DCW group. The Strict group was defined as a DCW $\leq 200 \%$ of the candidate's weight. The Liberal group was defined as a DCW $>200 \%$ of the candidate's weight. Baseline characteristics and outcomes were then compared between these 2 groups. A competing risk analysis, as well as a Kaplan-Meier survival curve were also performed for these groups.

\section{RESULTS}

\section{Overall}

A total of 7067 patients were included in the study. Minimum acceptable weight was normally distributed just below the candidate's actual weight with a mean of $81.1 \% \pm 17.2 \%$ of the patient's weight. Maximum acceptable DCW was right skewed and varied greatly with a median of $200 \%$ (range, $159.7 \%-241.4 \%$ ) of the candidate's weight. Furthermore, the fifth and 95th percentiles were $129 \%$ and $329 \%$ of the candidate's weight. The lowest acceptable DCW was reported to be $75 \%$ of the candidate's weight and the highest acceptable DCW was at $7400 \%$ of the candidate's weight. Figure E1 shows the maximum acceptable weight categorized by the candidate's actual weight. The maximum acceptable DCW decreased significantly with increasing candidate weight $(P<.001)$.

The baseline characteristics for the patient cohort are described in Table 1. Compared with the interquartile group, patients in the lower quartile were older (9 years; range, 3 15 years; $P<.001)$, with more VAD support $(243$ $[19.9 \%] ; P=.001)$ and less ECMO support (54 [4.4\%]; $P<.001)$ at transplant. They also were less frequently mechanically ventilated $(124[10.2 \%] ; P<.001)$ with better renal function $(190[15.9 \%] ; P<.001)$ at transplant and were more likely to have a diagnosis of cardiomyopathy 
TABLE 1. Patient characteristics and outcomes by donor to candidate weight ratio group (Group A: Lower 25\%, Group B: $25 \%-75 \%$, Group C: Upper $75 \%$ )

\begin{tabular}{|c|c|c|c|c|c|}
\hline Variable* & Group B $(\mathbf{n}=3529)$ & Group A $(n=1763)$ & $\begin{array}{c}\text { A vs B } \\
P \text { value }\end{array}$ & Group C $(n=1775)$ & $\begin{array}{l}B \text { vs } C \\
P \text { value }\end{array}$ \\
\hline Age (y) & $5(0-11)$ & $9(3-15)$ & $<.001$ & $3(0-6)$ & $<.001$ \\
\hline Female gender & $45.2(1595)$ & $42.9(755)$ & .109 & $46.1(819)$ & .517 \\
\hline Weight (kg) & $23(6-36)$ & $40(14-60)$ & $<.001$ & $14(4-18)$ & $<.001$ \\
\hline Listing max weight of patient weight (\%) & $145(130-151)$ & $200(185-204)$ & $<.001$ & $288(259-313)$ & $<.001$ \\
\hline \multicolumn{6}{|l|}{ Diagnosis } \\
\hline Cardiomyopathy & $44.6(1574)$ & $50.9(898)$ & $<.001$ & $38.2(678)$ & $<.001$ \\
\hline CHD & $46.8(1653)$ & 35.7 (629) & $<.001$ & $54.6(969)$ & $<.001$ \\
\hline Retransplant & $6.0(211)$ & $10.2(179)$ & $<.001$ & $4.5(79)$ & .021 \\
\hline Other & $2.3(91)$ & $3.2(57)$ & .152 & $2.8(49)$ & .590 \\
\hline \multicolumn{6}{|l|}{ Race } \\
\hline White & $55.4(1956)$ & $51.3(904)$ & .004 & $56.7(1007)$ & .049 \\
\hline African American & $20.6(728)$ & $23.5(414)$ & .017 & $18.3(324)$ & .041 \\
\hline Hispanic & $17.7(625)$ & $18.9(334)$ & .271 & $18.0(319)$ & .729 \\
\hline Other & $6.3(219)$ & $6.3(111)$ & .888 & $7.1(125)$ & .244 \\
\hline VAD at listing & $7.0(247)$ & $9.5(168)$ & .001 & $4.8(85)$ & .002 \\
\hline VAD at transplant & $16.5(386 / 2335) \dagger$ & $19.9(243 / 1219) \dagger$ & .003 & $12.8(163 / 1273) \dagger$ & .048 \\
\hline Mechanical ventilation at listing & $25.9(914)$ & $17.1(301)$ & $<.001$ & $27.8(494)$ & .133 \\
\hline Mechanical ventilation at transplant & $19.0(441 / 1320) \dagger$ & $10.2(124 / 1214) \dagger$ & $<.001$ & $23.8(301 / 1267) \dagger$ & $<.001$ \\
\hline ECMO at listing & $11.7(414)$ & $8.3(147)$ & $<.001$ & $11.5(204)$ & .798 \\
\hline ECMO at transplant & $6.0(139 / 2320) \dagger$ & $4.4(54 / 1148) \dagger$ & .050 & $8.4(107 / 1267) \dagger$ & .006 \\
\hline $\mathrm{eGFR}<60 \mathrm{~mL} / \mathrm{min} / 1.73 \mathrm{~m}^{2}$ at listing & $28.6(947 / 3315) \dagger$ & $19.9(340 / 1705) \dagger$ & $<.001$ & $33.6(548 / 1632) \dagger$ & $<.001$ \\
\hline $\mathrm{eGFR}<60 \mathrm{~mL} / \mathrm{min} / 1.73 \mathrm{~m}^{2}$ at transplant & $19.9(457 / 2294) \dagger$ & $15.9(190 / 1194) \dagger$ & .004 & $25.8(324 / 1254) \dagger$ & $<.001$ \\
\hline PRA $>10 \%$ & $18.2(642 / 1979) \dagger$ & $20.4(360 / 1094) \dagger$ & .792 & $16.9(301 / 1013) \dagger$ & .129 \\
\hline Ischemic time (h) & $3.5(2.9-4.2)$ & $3.5(2.8-4.2)$ & .377 & $3.7(3.1-4.5)$ & $<.001$ \\
\hline Days on waitlist & $39(14-97)$ & $52(16-147)$ & $<.001$ & $38(13-87)$ & .118 \\
\hline Offers/candidate/month & $5.6(1.3-6.4)$ & $5.0(0.9-5.9)$ & $<.001$ & $7.2(2.0-8.3)$ & $<.001$ \\
\hline Transplant rate & $66.2(2335)$ & $69.1(1219)$ & .030 & $71.7(1273)$ & $<.001$ \\
\hline Adverse outcome on waitlist & $20.5(729)$ & $15.0(266)$ & $<.001$ & $18.5(328)$ & .061 \\
\hline Actual donor-to-recipient weight (\%) & $132(107-161)$ & $110(95-125)$ & $<.001$ & $161(121-207)$ & $<.001$ \\
\hline
\end{tabular}

Values are presented as median (interquartile range) or \% (n). CHD, Congenital heart disease; $V A D$, ventricular assist device; $E C M O$, extracorporeal membranous oxygenation; $e G F R$, estimated glomerular filtration rate; $P R A$, panel reactive antibody. *For at-transplant variables: Group A: $\mathrm{n}=1219$, Group B: $\mathrm{n}=2335$, Group C: $\mathrm{n}=1273$. $\dagger$ Missing data, so totals are reported in parentheses.

(898 [50.9\%]; $P<.001)$. Conversely, the upper quartile group was younger compared with the interquartile group (3 years [range, $0-6$ years]; $P<.001$ ) and more frequently had a diagnosis of CHD $(969[54.6 \%] ; P<.001)$. They were also more frequently mechanically ventilated (301 [23.8\%]; $P<.001)$ with more renal impairment $(324$ $[25.8 \%] ; P<.001)$ and ECMO support (107 [8.4\%]; $P=.006)$ at transplant.

Figure 1 shows how median DCW varies by different institutions across the United States. for those centers that performed 20 or more transplants during the study period. Despite large differences in listing habits, the transplanted DCW was tightly clustered around $130 \%$.
Waitlist outcomes and posttransplant outcomes are described in Table 1. The transplant rate of both the upper quartile $(1273[71.7 \%] ; P<0.001)$ and lower quartile $(1219[69.1 \%] ; P=.030)$ was significantly greater than the interquartile group (2335 [66.2\%]). The lower quartile group had the best waitlist survival (Figure 2), despite having longer waitlist times (52 days [range, 16-147 days]; $P<.001)$ with the fewest adverse events (266 [15.0\%]; $P<.001)$. The number of organ offers each candidate received per month increased from 5.0 in the lower quartile to 5.6 in the interquartile to 7.2 for the upper quartile (all $P$ values $<.001$. Accordingly, actual transplant DCW also increased from $110 \%$ in the 


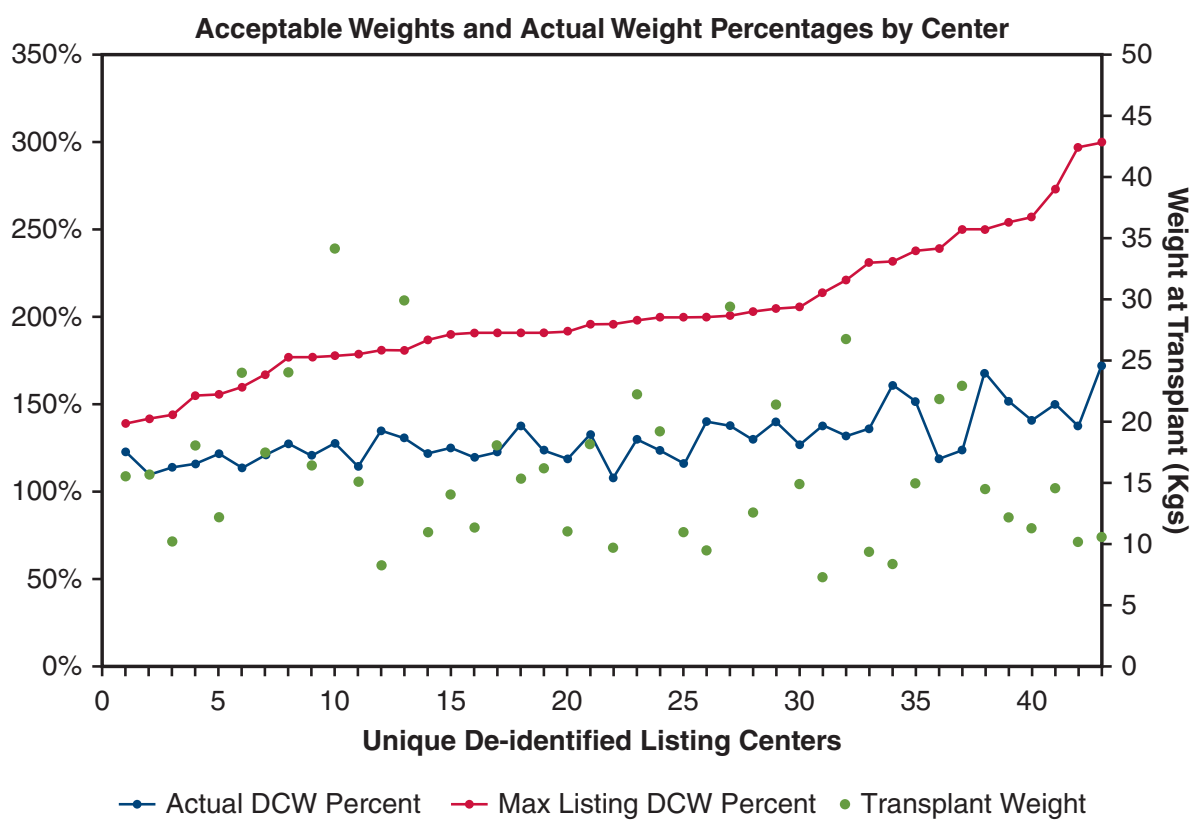

FIGURE 1. Median institutional maximal acceptable listing donor-candidate weight ratio $(D C W)(r e d)$ and accepted donor-to-recipient transplants ratios (blue) and median transplanted patient weight (green). Despite maximal listing DCW ranging from $141 \%$ to $298 \%$, most centers match to donors between $110 \%$ and $140 \%$ of the patient's weight.

lower quartile to $132 \%$ in the middle quartile to $161 \%$ in the upper quartile.

On multivariable analysis controlling for known risk factors, DCW (hazard ratio [HR], 0.939; 95\% confidence interval $[\mathrm{CI}], 0.820-1.073 ; P=.365)$ was not associated with waitlist survival (Table 2). ECMO at listing was the strongest predictor of an adverse event (HR, 3.614, 95\% CI, 3.109-4.201; $P<.001$ ), whereas VAD at listing was the only protective factor (HR, $0.732 ; 95 \% \mathrm{CI}, 0.580$ $0.922 ; P=.008$ ) on the waitlist.

While on the waitlist, $37.2 \%$ of patients had their maximal acceptable DCW altered, and $29.9 \%$ had it altered by $>10 \%$ of their starting weight. Changes in acceptable donor weight while listed more frequently occurred in infants $(44.8 \%$ vs $33.1 \% ; P<.001)$ and those who were on the waitlist for longer periods of time $(P<.001)$ (Figure E2). This ranged from 0 to 14 changes in acceptable weight. When examining all changes, the median change was an increase of $25.0 \%$ (range, $13.5 \%-47.9 \%$ ) from the previous listing.

Of 4827 patients undergoing transplant, the median accepted DCW was $127 \%$ (range, 105\%-161\%). The largest actual DCW was $408 \%$ of recipient weight and the smallest was 50\%. Posttransplant survival was similar for the 3 categories (Figure E3). The lower quartile group had the shortest median posttransplant length of stay at 16 days (range, 11-28 days) compared with the interquartile group (18 days [range, 12-31 days]; $P<.001)$ and upper quartile group (19 days [range,
12-34 days]; $P<.001)$. The upper and interquartile groups were similar $(P=.740)$.

\section{Critically Ill Children}

In the subgroup analysis of critically ill children who received mechanical ventilation, had renal dysfunction, and had CHD, the overall median maximum acceptable DCW for the whole group was 205\% (range, 190\%$275 \%$ ) with similar baseline characteristics (Table 3). The Liberal group received more offers per month and was associated with more patients being transplanted and fewer adverse events while on the waitlist. They had better waitlist survival by Kaplan-Meier (Figure 3) curve analysis $(P=.005)$, which was further evaluated by a competing outcomes analysis. Competing outcomes demonstrated that most of the events took place during the first 2 months after listing for transplant (Figure 4). The Liberal group had $42 \%$ of patients undergo transplant, whereas only $36 \%$ died waiting, compared with $30 \%$ transplanted with $53 \%$ dying on the waitlist in the Strict group. The 2 groups experienced similar posttransplant survival regardless of maximum listing weight criteria (Figure E4).

\section{DISCUSSION}

We identified significant practice variability in the weight range criteria used when listing a pediatric patient for heart transplantation. This is suspected to be due to the lack of accessible methods for accurately assessing heart size, as well as a paucity of studies demonstrating heart size 


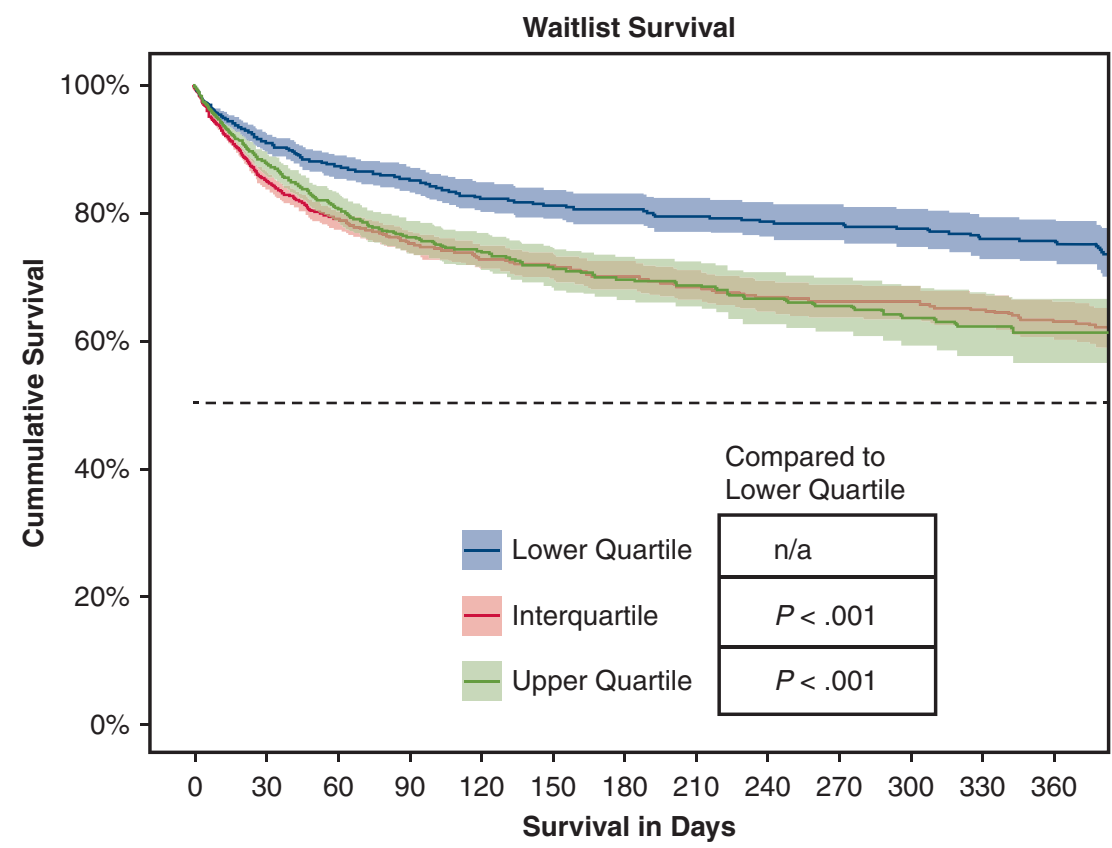

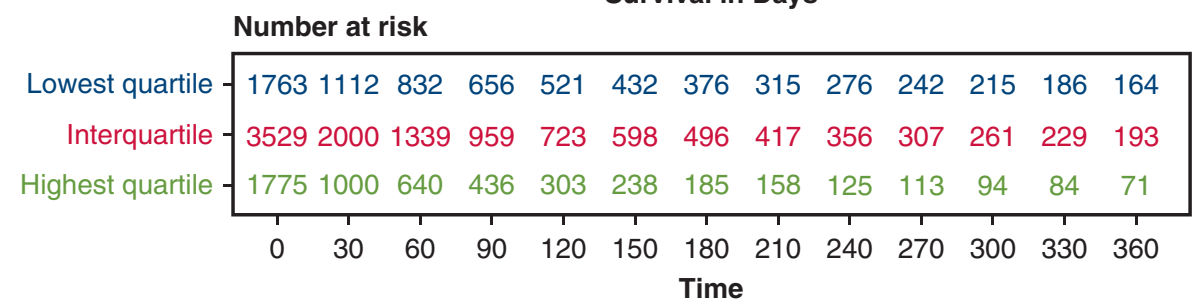

FIGURE 2. Waitlist survival of patients grouped by their maximal acceptable donor-candidate weight ratio. Patients in the lower quartile demonstrated superior weight list survival to the intequartile and upper quartile groups. $n / a$, Not available.

mismatch affecting survival after pediatric heart transplant. Despite this practice variability, there was no difference in posttransplant survival between groups listed with different weight ranges-even when they accepted organs from donors of significantly different sizes. Prior studies have revealed conflicting results regarding the influence of donor-to-recipient weight ratio on survival. Literature

TABLE 2. Multivariable analysis of waitlist survival

\begin{tabular}{lcr}
\hline \multicolumn{1}{c}{ Variable } & $\begin{array}{c}\text { Hazard ratio }(95 \% \\
\text { confidence interval) }\end{array}$ & $\begin{array}{c}\boldsymbol{P} \\
\text { value }\end{array}$ \\
\hline $\begin{array}{l}\text { DCW } 25 \%-75 \% \text { compared with } \\
<25 \%\end{array}$ & $0.939(0.820-1.076)$ & .365 \\
DCW $>75 \%$ compared with $<25 \%$ & $1.013(0.873-1.175)$ & .866 \\
\hline Diagnosis of CHD & $1.902(1.688-2.143)$ & $<.001$ \\
\hline Mechanical ventilation at listing & $2.228(1.948-2.547)$ & $<.001$ \\
\hline ECMO at listing & $3.614(3.109-4.201)$ & $<.001$ \\
eGFR $<60 \mathrm{~mL} / \mathrm{min} / 1.73 \mathrm{~m}^{2}$ at listing & $2.199(1.947-2.482)$ & $<.001$ \\
\hline VAD at listing & $0.732(0.580-0.922)$ & .008 \\
\hline
\end{tabular}

$\overline{D C W}$, Donor to candidate weight ratio; $C H D$, congenital heart disease; $E C M O$, extracorporeal membranous oxygenation; $e G F R$, estimated glomerular filtration rate; $V A D$, ventricular assist device. from adult cardiac transplantation suggests donor undersizing helps increase the donor pool, ${ }^{11}$ and a UNOS database review showed that weight ratio did not affect survival. ${ }^{2}$ Although limited, most pediatric studies discourage donor undersizing and show a survival benefit to oversizing ${ }^{12-14}$ or no difference in posttransplant outcomes, ${ }^{7}$ which is similar to our analysis. Unfortunately, these studies are mostly single-center experiences and do not expand on many other factors that could account for their findings, leaving uncertainty and skepticism amongst transplant centers.

In our large, multicenter analysis, listing patients with different maximal DCW did not affect waitlist or posttransplant survival, except in the critically ill subset. In addition, factors that one may surmise would influence waitlist DCW in children, such as diagnosis, did not seem to have an effect. For example, one might expect a patient with dilated cardiomyopathy to have a thoracic cavity capable of accommodating a heart from a much larger donor. For this reason, dilated cardiomyopathy candidates were excluded from prior studies examining weight ratios in transplant. ${ }^{7,14}$ Our analysis paradoxically showed that greater maximal acceptable weight is actually used in patients with CHD 
TABLE 3. Baseline demographic characteristics and outcome data for subanalysis of critically ill children

\begin{tabular}{|c|c|c|c|}
\hline Variable & $\begin{array}{c}\text { Strict weight } \\
\text { group DCW } \\
<\mathbf{2 0 0} \% \\
(\mathbf{n}=\mathbf{2 1 5})\end{array}$ & $\begin{array}{c}\text { Liberal weight } \\
\text { group DCW } \\
\geq \mathbf{2 0 0} \% \\
(\mathbf{n}=\mathbf{2 4 7})\end{array}$ & $\begin{array}{c}P \\
\text { value }\end{array}$ \\
\hline \multicolumn{4}{|l|}{ Baseline } \\
\hline Age at listing (mo) & $0(0-4)$ & $0(0-3)$ & .146 \\
\hline Female gender & $37(80)$ & $39(95)$ & .077 \\
\hline African American race & $13(27)$ & $21(51)$ & .021 \\
\hline Weight $(\mathrm{kg})$ & $4.0(3.2-6.0)$ & $3.8(3.2-5.5)$ & .174 \\
\hline ECMO at transplant & $25(21)$ & $24(31)$ & .861 \\
\hline VAD at transplant & $1.9(4)$ & $1.6(4)$ & .843 \\
\hline Listing max DCW (\%) & $188(165-199)$ & $259(222-300)$ & $<.001$ \\
\hline \multicolumn{4}{|l|}{ Outcomes } \\
\hline Offers per month & $2.31(0-4.4)$ & $3.21(1.5-6.7)$ & $<.001$ \\
\hline Days on waitlist & $25(10-61)$ & $23(9-51)$ & .203 \\
\hline Adverse events & $53(113)$ & $39(96)$ & .003 \\
\hline Received transplant & $40(85)$ & $53(131)$ & .003 \\
\hline Donor age $(\mathrm{mo})$ & $4(2-11)$ & $5(2-20)$ & .579 \\
\hline $\begin{array}{l}\text { Donor to recipient weight } \\
\text { ratio }(\%)\end{array}$ & $142(117-166)$ & $164(125-215)$ & .153 \\
\hline
\end{tabular}

Values are presented as median (interquartile range) or \% (n). DCW, Donorcandidate weight ratio; $E C M O$, extracorporeal membranous oxygenation; $V A D$, ventricular assist device.

compared with cardiomyopathy. Therefore, transplant teams may be adjusting waitlist criteria to increase offers in patients who are more ill or at higher risk. In line with that thought process, a maximal acceptable weight closer to a patients' actual weight was more commonly seen when patients were less ill. This is supported by the decreased incidence of ECMO, mechanical ventilation, and renal dysfunction both at listing and transplant in the lower quartile. Their overall lower burden of illness appeared to contribute to their superior waitlist survival on multivariable analysis, and their listing DCW ratio was not found to be significant. All this is despite the lower quartile having longer waitlist times and a lower proportion of the group being transplanted at the end of the study period. The lower quartile had the greatest percentage of cardiomyopathy diagnoses that may represent the greatest potential for recovery or lower acuity. Accordingly, the upper quartile had the most CHD diagnoses as well as the smallest children. Additionally, it was associated with more mechanical ventilation at transplant and worse renal function at listing and at transplant. This perhaps represents a group with more growth potential, a willingness to accept a larger organ, and an increased urgency to find a suitable donor.

While only one third of patients had their maximum DCW change while on the waitlist, this occurred more often in smaller patients and those on the waitlist longer. This may represent growth in these patients while listed or potentially a need to elicit more offers. In all, we were surprised to find such considerable differences between the groups of patients listed with different acceptable DCWs. It may be that certain centers use differing acceptable DCWs for different patient populations in addition to having differences in practice preferences among centers. Although our main focus was on patient-level outcomes, it is also possible that higher volume centers would have more high-risk children. That fact, along with their greater experience, may affect listing patterns.

The primary differences in outcomes between the groups were the number of offers per month, the amount of time spent on a waitlist, and the actual DCW at transplant. The upper quartile received the most offers, whereas the lower quartile had the longest wait times. Logically, the actual transplanted DCW increased with higher quartiles. These are key findings of this study because prior works only focused on posttransplant outcomes. It is important to note that waitlist attrition can be high and represents an important time where patients may either be optimized for transplant or deteriorate into Interagency Registry for Mechanically Assisted Circulatory Support level I status. However, we found that DCW does not affect this survival. Whether or not the differences in listing habits across the country are influencing the quality of care patients receive on a center-by-center basis is unclear. Yet, there is certainly a degree of disparity generated if a patient who would have been listed with a higher acceptable weight at 1 center is listed at another center with a lower limit and, therefore, receives fewer offers. A multitude of variables and clinical decision making ultimately result in the listing patterns, yet further emphasis on waitlist outcomes and donor offer acceptance patterns may help improve outcomes for all patients. Davies and colleagues ${ }^{15}$ recently demonstrated the mortality risk of refusing even 1 offer on the waitlist is $15 \%$.

Despite the heterogeneity among the groups, these practices led to posttransplant survival that was remarkably similar amongst the groups. This finding raises the question of how the upper quartile, a group of patients with more CHD, mechanical ventilation, and renal dysfunction, can have comparable survival to members of the lower quartile, which was composed of older patients who more commonly had cardiomyopathy, had more VAD use, and had fewer other risk factors for posttransplant mortality. Perhaps the beneficial result of using a larger organ $(161 \%$ vs $110 \%$ of a patient's weight) is masked by the apparent need to expand the weight range criteria in sicker patients in an attempt to find a suitable donor sooner.

With this in mind, we identified a group of 462 critically ill patients who were at high risk for mortality. A very liberal listing criteria weight range ( $>200 \%$ recipient weight) was associated with fewer adverse events while on the waitlist, more offers, and a higher rate of transplant. However, posttransplant survival was comparable. Although posttransplant survival did not differ, the more liberal weight group 


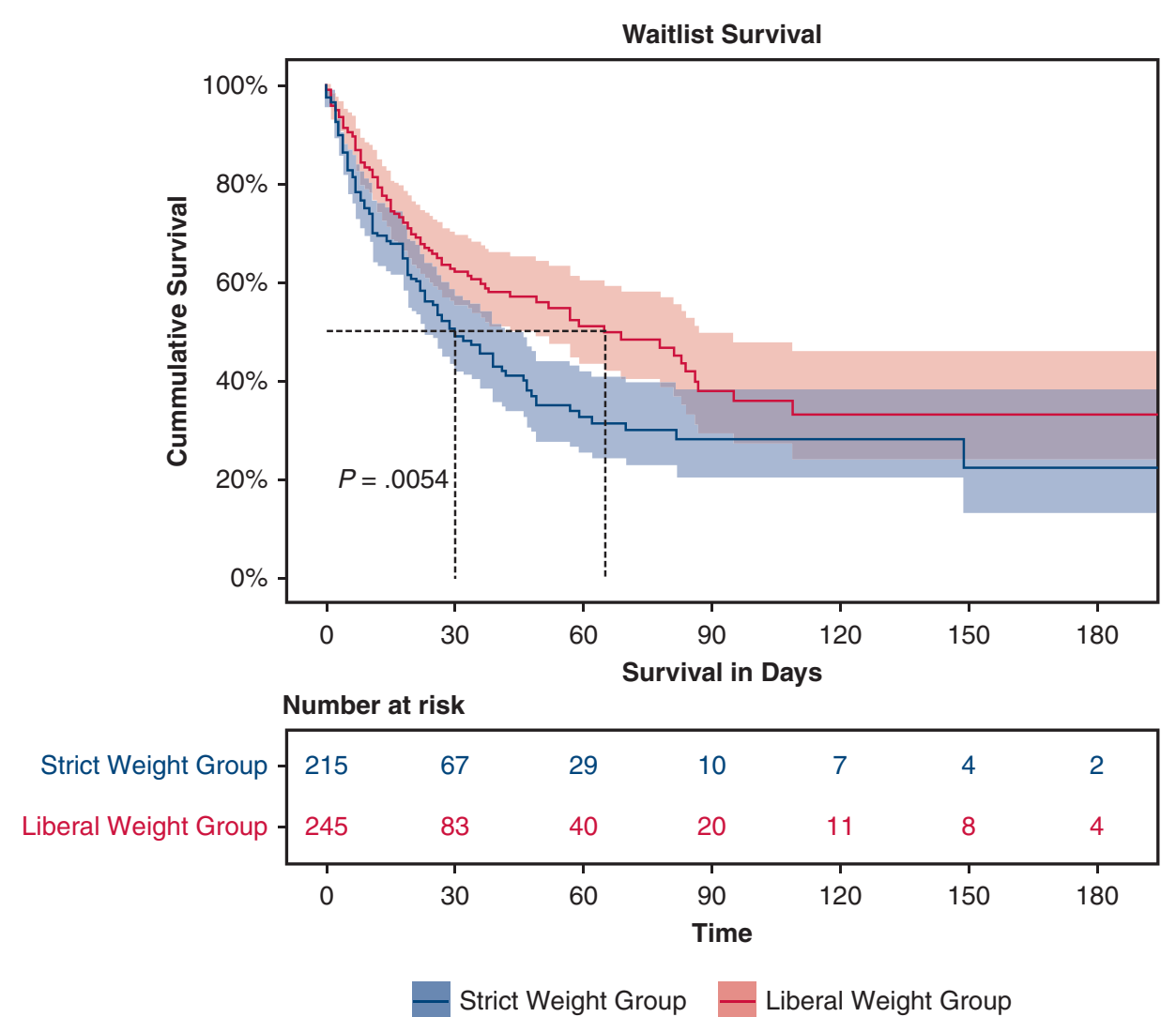

FIGURE 3. Kaplan-Meier curve showing superior waitlist survival for critically ill children listed in the Liberal weight group.

benefited by having superior waitlist survival, even with similar risk factors for mortality. Therefore, they experienced an overall better survival from day of listing. From this, we conclude that these critically ill infants who have not been separately analyzed before may be an exception to the rest, in which case utilizing what many may consider an excessively large weight range may lead to superior outcomes.

Lastly, these data suggest that we reassess the criteria upon which we have been turning down pediatric cardiac donors because donor-to-recipient weight ratio only appeared to influence waitlist time with no difference in survival after transplant. If $34 \%$ of donor hearts do not get utilized due to size or weight mismatch, ${ }^{8}$ our findings may represent an opportunity to broaden the donor pool. This could increase the number of pediatric heart transplants performed annually while decreasing wait time without increasing morbidity or mortality. A single-center study from the United Kingdom reported comparable outcomes in patients of all weight ratios with a policy of accepting up to $300 \%$ of their candidates' weight to help maximize donor organ use. ${ }^{14} \mathrm{~A}$ technology that may help alleviate some of the angst associated with weight mismatch is the use of virtual transplantation, whereby using computed tomography or magnetic resonance imaging data cardiac volume can be estimated for both the donor and the candidate to determine the exact fit. This technology has already helped to expand use of mechanical circulatory support devices and is currently being evaluated in transplantation. ${ }^{16,17}$ Even with this technology, there would be a range of sizes that could be made to fit within a candidate's thoracic cavity. This could allow a potentially greater pool of donors to be identified for each candidate on the waitlist with a sound, reproducible, scientific basis and reduced disparity. This would also allow for the investigation of the influence of predicted heart mass because this metric has been recently advocated for in the adult literature. ${ }^{18}$

\section{Limitations}

Limitations of our study are those typical of a retrospective review because we used the UNOS database. Although associations are present, no causal effects can be defined among listing weights and patient outcomes. The primary issue is that we cannot discern the actual complex decision-making process that led to the selected weight ranges for each patient. Also, with a limited number of predefined variables collected at specific times, we may be 


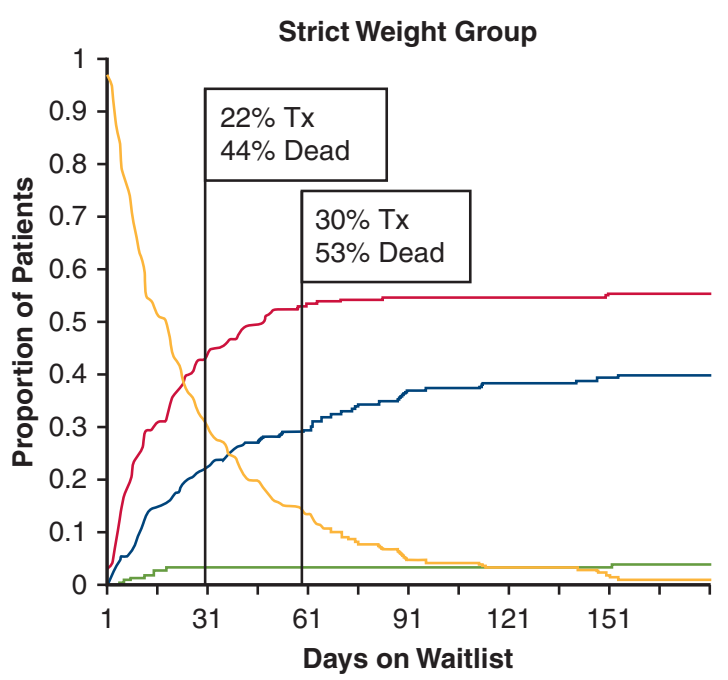

A

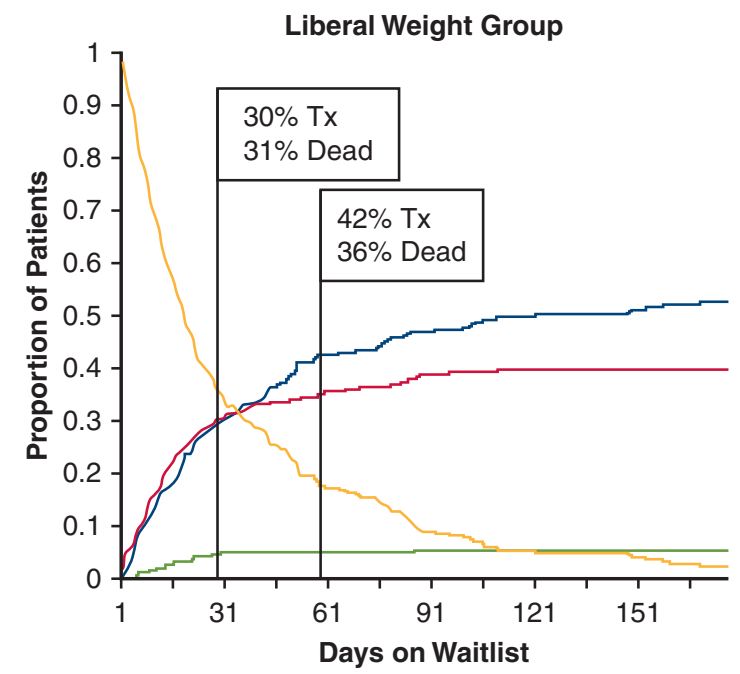

B

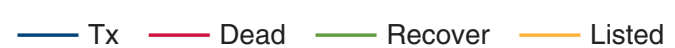

FIGURE 4. Competing outcomes analysis of waitlist survival. A, Strict weight group. B, Liberal weight group. Indicators of 1- and 2-month outcomes show that in both groups, $>77 \%$ of patients have either received transplanted $(T x)$ or died within the first 2 months, making the acceptance of early offers critical to avoiding waitlist mortality.

missing a key factor that affected the maximum weight chosen. Lastly, it must be kept in mind that patients with CHD, and to some degree dilated cardiomyopathy, are a heterogeneous group that is not further well classified within this dataset.

\section{CONCLUSIONS}

In all, we found large variation in the weight range set forth for individual patients. Furthermore, there was vast intercenter variation in median acceptable donor weight, despite finding no influence on waitlist or posttransplant survival. Therefore, the utility of waitlist weight criteria in pediatric cardiac transplantation should be reconsidered, as well as possibly normalizing the criteria programs use, or including supplemental methods to size-match analysis. Although a more liberal weight range criteria was found to benefit select critically ill patients, arbitrarily acceptable weight range restrictions seem unjustified in most pediatric candidates based on our data. If better consensus among programs was achieved, we could potentially improve equality of organ allocation, and the overall pediatric cardiac transplant candidate pool could prosper from better donor organ use.

\section{Conflict of Interest Statement}

Authors have nothing to disclose with regard to commercial support.

\section{References}

1. Heart size estimations in adults. Acta Radiol. 1950;33(82 Suppl):11-29.

2. Patel ND, Weiss ES, Nwakanma LU, Russel SD, Baumgartner WA, Shah AS, et al. Impact of donor-to-recipient weight ratio on survival after heart transplantation: analysis of the United Network for Organ Sharing Database. Circulation. 2008;118:S83-8.

3. Reed RM, Netzer G, Hunsicker L, Mitchell BD, Rajagopal K, Scharf S, et al. Cardiac size and sex-matching in heart transplantation: size matters in matters of sex and the heart. JACC Heart Fail. 2014;2:73-83.

4. Patel A, Bock MJ, Wollstein A, Nguyen K, Malerba S, Lytrivi ID. Donor-recipient height ratio and outcomes in pediatric heart transplantation. Pediatr Trans plant. 2016;20:652-7.

5. Barkoff LM, Maeda K, Rosenthal DN, Zhang Y, Hollander SA, Dykes JC, et al. Center variation in listing parameters among US pediatric heart transplant programs. J Heart Lung Transplant. 2018;37:S396.

6. Tang L, Du W, Delius RE, L'Ecuyer TJ, Zilberman MV. Low donor-to-recipient weight ratio does not negatively impact survival of pediatric heart transplant patients. Pediatr Transplant. 2010;14:741-5.

7. Razzouk AJ, Johnston JK, Larsen RL, Chinnock RE, Fitts JA, Bailey LL. Effect of oversizing cardiac allografts on survival in pediatric patients with congenital heart disease. J Heart Lung Transplant. 2005; 24:195-9.

8. Rizwan R, Zafar F, Bryant R, Tweddell JS, Lorts A, Chin C, et al. The number of refusals for donor organ quality does not impact heart transplant outcomes in children. Ann Thorac Surg. 2018;105:1223-30.

9. Morrison AK, Gowda C, Tumin D, Phelps CM, Hayes D, Tobias J, et al. Pediatric marginal donor hearts: trends in US national use, 2005-2014. Pediatr Transplant. 2018;22:e13216.

10. Davies RR, Russo MJ, Mital S, Martens TM, Sorabella RS, Hong KN, et al. Predicting survival among high-risk pediatric cardiac transplant recipients: an analysis of the United Network for Organ Sharing database. J Thorac Cardiovasc Surg. 2008;135:147-55.

11. Costanzo-Nordin MR, Liao YL, Grusk BB, O'Sullivan EJ, Cooper RS, Johnson MR, et al. Oversizing of donor hearts: beneficial or detrimental? J Heart Lung Transplant. 1991;10:717-30.

12. Tamisier D, Vouhé P, Le Bidois J, Mauriat P, Khoury W, Leca F. Donor-recipien size matching in pediatric heart transplantation: a word of caution about small grafts. J Heart Lung Transplant. 1996;15:190-5.

13. Tjang YS, Stenlund H, Tenderich G, Hornik L, Bairaktaris A, Körfer R. Risk fac tor analysis in pediatric heart transplantation. J Heart Lung Transplant. 2008;27: 408-15.

14. Kanani M, Hoskote A, Carter C, Burch M, Tsang V, Kostolny M. Increasing donor-recipient weight mismatch in pediatric orthotopic heart transplantation does not adversely affect outcome. Eur J Cardiothorac Surg. 2012;41: 427-34.

15. Davies RR, Bano M, Butts RJ, Jaquiss RDB, Kirk R. Donor organ turn-downs and outcomes after listing for pediatric heart transplant. J Heart Lung Transplant. 2019;38:241-51. 
16. Moore RA, Lorts A, Madueme PC, Taylor MD, Morales DLS. Virtual implantation of the 50cc SynCardia total artificial heart. J Heart Lung Transplant. 2016; 35:824-7.

17. Moore RA, D'Souza GA, Villa C, Taylor MD, Morales DLS, Lorts A. Optimizing surgical placement of the HeartWare ventricular assist device in children and adolescents by virtual implantation. Prog Pediatr Cardiol. 2017;47: 11-3.
18. Kransdorf EP, Kittleson MM, Benck LR, Patel JK, Chung JS, Esmailian F, et al Predicted heart mass is the optimal metric for size match in heart transplantation. J Heart Lung Transplant. 2019;38:156-65.

Key Words: pediatric, heart failure, cardiac transplantation, waitlist, weight 


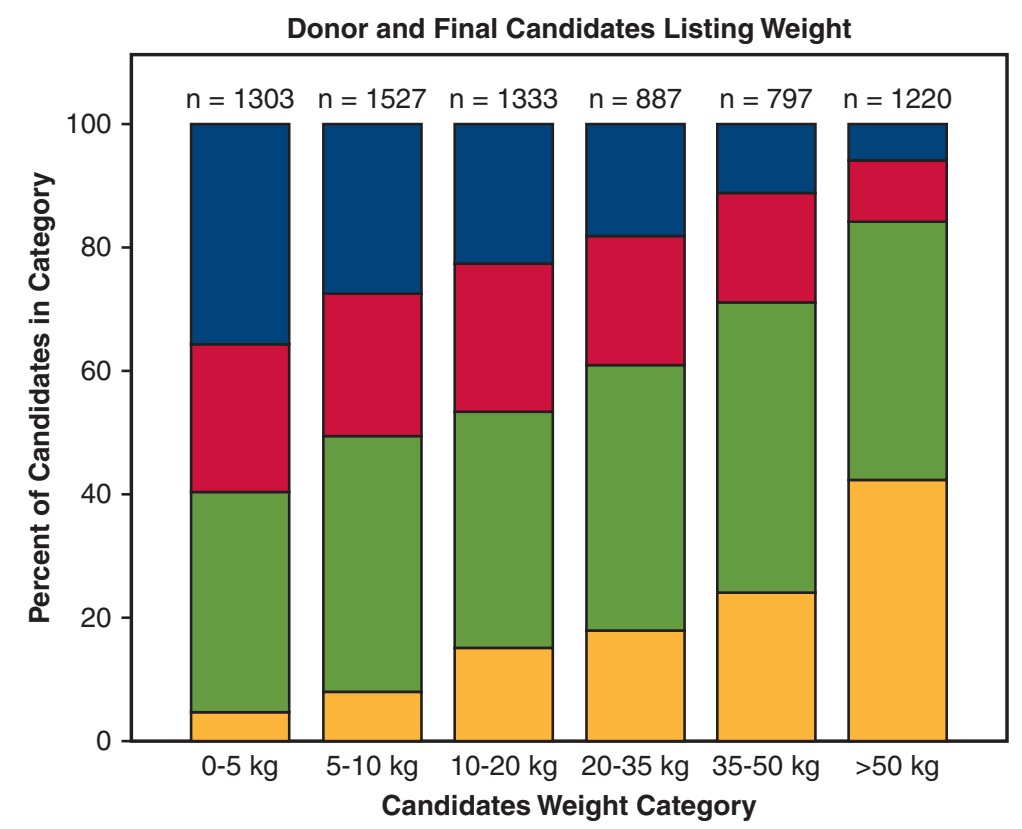

Maximum acceptable weight percent of candidates

$\square>250 \% \quad \square 200-250 \% \quad \square 150-200 \% \quad \square<150 \%$

FIGURE E1. The proportion of patients in 4 different ranges of maximum acceptable donor-candidate weight ratio. There is a trend toward accepting a larger donor in smaller children, whereas a candidate closer to the patient's size is more desirable in children $>50 \mathrm{~kg}$. Candidates are grouped by their final weight. 


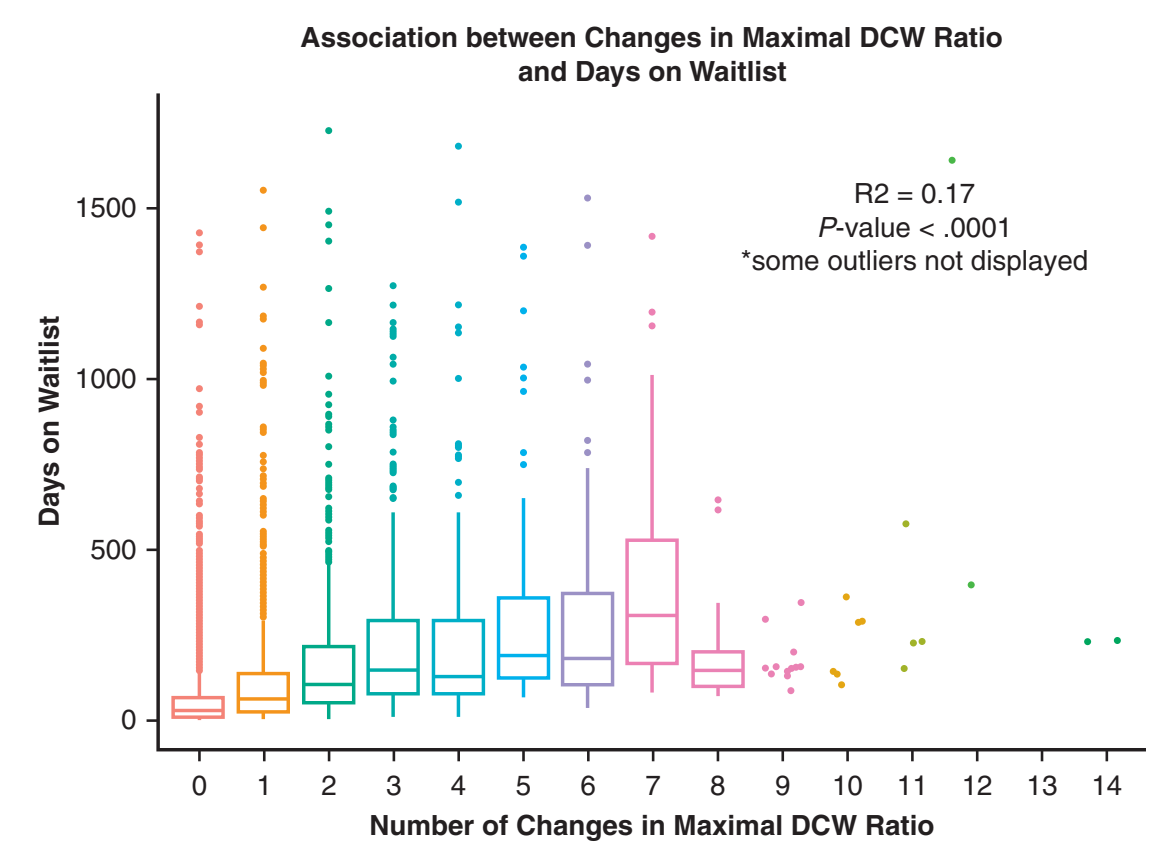

\begin{tabular}{|c|c|c|c|c|c|c|c|c|c|c|c|c|c|c|c|}
\hline $\mathrm{N}$ & 4441 & 1451 & 583 & 281 & 139 & 61 & 41 & 27 & 17 & 11 & 6 & 5 & 2 & & 2 \\
\hline Median & 25 & 60 & 102 & 146 & 126 & 186 & 177 & 308 & 142 & 153 & 214 & 229 & 1018 & & 232 \\
\hline
\end{tabular}

FIGURE E2. Box and whiskers plot showing that as the number of changes in maximal donor-candidate weight ratio $(D C W)$ increase, so does number of days on the waitlist such that patients waiting for an organ longer are likely to have more changes in their acceptable DCW. The upper and lower borders of the box represent the upper and lower quartiles. The middle horizontal line represents the median. The upper and lower whiskers represent the maximum and minimum values of nonoutliers. Extra dots represent outliers. If the number of subjects or measurements is $<15$, then each data point is plotted individually as a dot. 


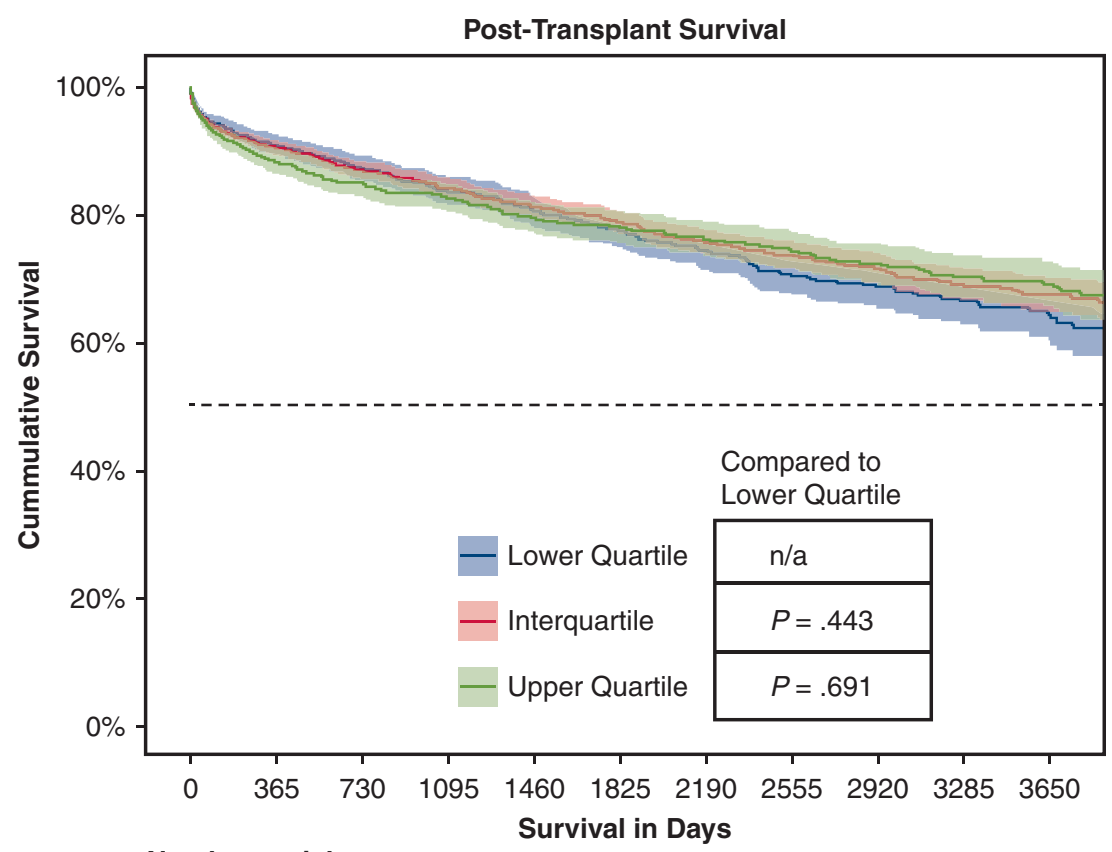

\begin{tabular}{|c|c|c|c|c|c|c|c|c|c|c|c|}
\hline \multirow[b]{2}{*}{ Lowest Quartile } & \multicolumn{11}{|c|}{ Number at risk } \\
\hline & 1214 & 949 & 800 & 683 & 562 & 445 & 341 & 268 & 202 & 141 & 95 \\
\hline Interquartile & 2321 & 1831 & 1563 & 1327 & 1107 & 925 & 747 & 599 & 466 & 345 & 237 \\
\hline \multirow[t]{2}{*}{ Highest Quartile } & 1267 & 964 & 797 & 683 & 575 & 488 & 409 & 335 & 281 & 218 & 161 \\
\hline & 0 & 365 & 730 & 1095 & 1460 & 1825 & 2190 & 2555 & 2920 & 3285 & 3650 \\
\hline
\end{tabular}

FIGURE E3. Posttransplant survival of patients grouped by their maximal acceptable donor-candidate weight ratio. There is no difference in posttransplant survival among the groups. $n / a$, Not available. 


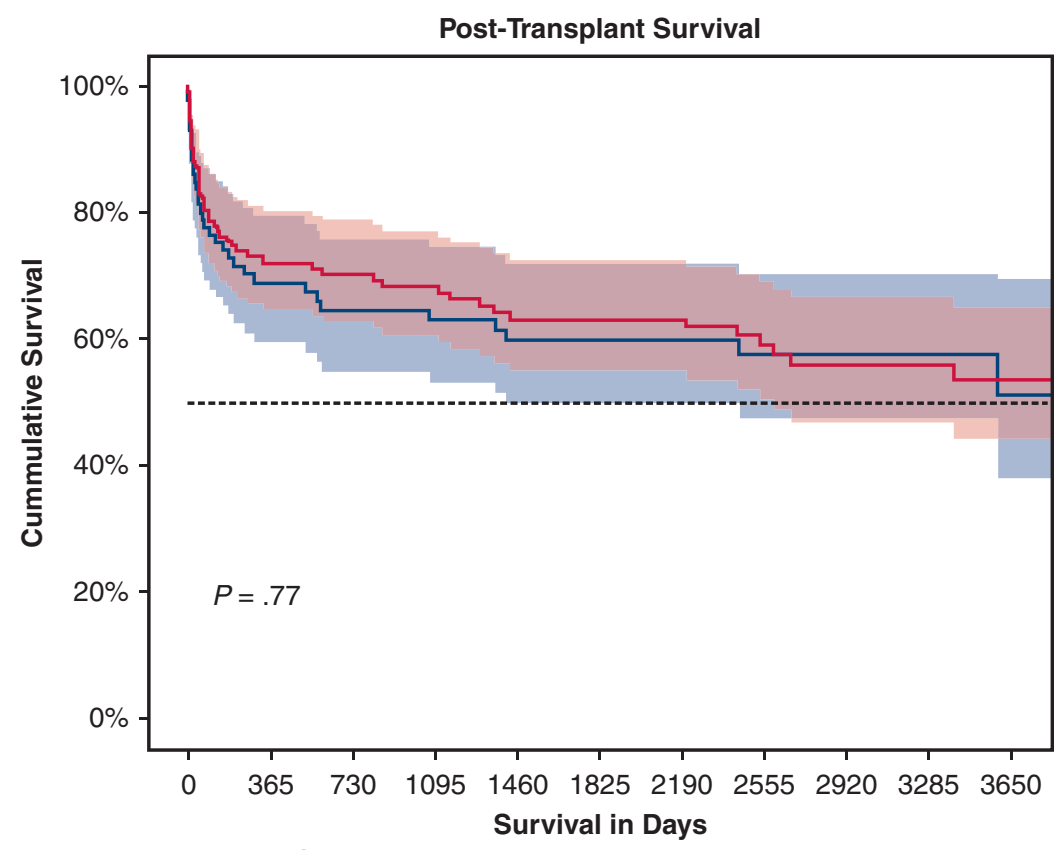

\begin{tabular}{|c|c|c|c|c|c|c|c|c|c|c|c|}
\hline \multirow[b]{2}{*}{ Strict Weight Group } & \multicolumn{11}{|c|}{ Number at risk } \\
\hline & 85 & 49 & 44 & 41 & 37 & 35 & 28 & 22 & 16 & 10 & 8 \\
\hline \multirow[t]{3}{*}{ Liberal Weight Group } & 131 & 85 & 75 & 69 & 59 & 54 & 49 & 39 & 33 & 29 & 25 \\
\hline & 0 & 365 & 730 & 1095 & 1460 & $\begin{array}{l}1825 \\
\text { Time }\end{array}$ & 2190 & 2555 & 2920 & 3285 & 3650 \\
\hline & & & St & trict We & eight $\mathrm{Gr}$ & roup & Lib & eral We & eight $\mathrm{G}$ & iroup & \\
\hline
\end{tabular}

FIGURE E4. Kaplan-Meier curve showing similar posttransplant survival for Liberal and Strict weight groups. 